\title{
Regulation of Runx 2 by microRNA-9 and microRNA-10 modulates the osteogenic differentiation of mesenchymal stem cells
}

\author{
HONG LUO $^{1}$, HUALIN GAO ${ }^{2}$, FANG LIU ${ }^{1}$ and BING QIU ${ }^{1}$ \\ ${ }^{1}$ Department of Orthopaedic Surgery, Guizhou Orthopaedics Hospital; ${ }^{2}$ Department of Anesthesiology, \\ Guizhou Orthopaedics Hospital, Guiyang, Guizhou 550001, P.R. China
}

Received May 6, 2016; Accepted January 24, 2017

DOI: $10.3892 / \mathrm{ijmm} .2017 .2918$

\begin{abstract}
Currently, the use of stem cell transplantation for the treatment of osteoporosis is a popular research topic. In general, promotion of the osteogenic differentiation of stem cells is the goal of researchers. MicroRNAs (miRNAs of miRs) are critical regulatory factors of osteogenic induction, and miR-9 and miR-10 have been substantiated to be involved in the osteogenic process, yet the underlying mechanisms remain unclear. The present study investigated the role of miR-9 and miR-10 in the osteogenic differentiation of bone marrow-derived mesenchymal stem cells (BMSCs) and aimed to elucidate the mechanism of osteogenic differentiation. Using MTT assay and RT-PCR, we found that miR-9 expression was upregulated and miR-10 expression was downregulated during osteogenic differentiation. Moreover, as shown by the results of mineralization analysis and western blot analysis, the overexpression of miR-9 enhanced the osteogenic abilities and overexpression of miR-9 inhibited these abilities. Furthermore, miR-9 and miR-10 also affected the expression of Runt-related transcription factor 2 (Runx2) and extracellular signal-regulated kinase (ERK) pathway by western blot analysis. Based on these results, we conclude that miR-9 and miR-10 play key roles in regulating the differentiation of BMSCs.
\end{abstract}

\section{Introduction}

Osteoporosis is a chronic non-communicable disease characterized by reduced bone mass and the disruption of bone architecture results in increased risk of fragility fractures. Osteoporosis has major impacts on individuals, healthcare systems and societies (1). The World Health Organization

Correspondence to: Mr. Hualin Gao, Department of Anesthesiology, Guizhou Orthopaedics Hospital, 123 ShaChong Road, Guiyang, Guizhou 550001, P.R. China

E-mail: hualin112-1@tom.com

Key words: microRNA-9, microRNA-10, osteogenic differentiation, Runt-related transcription factor 2, extracellular signal-regulated kinase
(WHO) considers osteoporosis to be next only to cardiovascular disease as a critical health issue (2). In China, the reported overall prevalence of osteoporosis based on nationwide surveys ranges from 6.6 to $19.3 \%$ (average, 13.0\%) (3). Moreover, it has been estimated by authority agency that the population with osteoporosis will increase sharply from 83.9 million in 1997 to 212 million by 2050 (4). For the prevention of osteoporosis, antiresorptive drugs (bisphosphonates and denosumab) that decrease high bone resorption and, osteoanabolic drugs (teriparatide) that promote bone formation, are the mainstream of therapy (5). The availability of antiresorptive and osteoanabolic drugs has enhanced our ability to reduce the risk of fracture. But, the efficacy of all of the drugs has not met our expectations. Increasing evidence suggests that senescence of functional stem cells is critical in aging and aging-associated degenerative diseases. As stem cells possess a high proliferative capacity and have the potential to differentiate into osteoblasts, stem cell transplantation is generally considered as a highly promising method for osteoporosis $(6,7)$. Mesenchymal stem cells are multipotent cells, which can replicate as undifferentiated cells and have the potential to differentiate into lineages of mesenchymal tissues, including bone, cartilage, fat, tendon, muscle and marrow stroma (8). Induction of the osteogenic differentiation of mesenchymal stem cells is the goal of the scientists, but the overall effect is still poor.

MicroRNAs (miRNAs or miRs) are endogenous 22-nt RNAs that can play important regulatory roles in animals and plants by targeting mRNAs for cleavage or translational repression (9). Of note, recent studies have demonstrated that miRNAs play significant roles in all stages of bone formation, suggesting the possibility that miRNAs can be novel therapeutic targets for skeletal diseases (10). In this study, we investigated the role and mechanism of action of miRNAs in the osteogenic differentiation of mesenchymal stem cells. Previous research has demonstrated that miR-9 and miR-10 play important roles in the differentiation of bone marrow-derived mesenchymal stem cells (BMSCs) $(11,12)$. Yet, it remains unclear whether miR-9 and miR-10 regulate osteogenic differentiation during the differentiation of BMSCs. Therefore, in this study, we sought to explore the role of miR-9 and miR-10 in BMSC osteogenic differentiation and to investigate the mechanisms of miR-9 and miR-10 in the promotion or inhibition of BMSC 
differentiation into osteoblasts. We hope that our research will contribute to the prevention of osteoporosis.

\section{Materials and methods}

Reagents. Fetal bovine serum (FBS), $\alpha$-minimal essential medium $(\alpha-\mathrm{MEM})$, penicillin and streptomycin were obtained from Invitrogen (Carlsbad, CA, USA). $\beta$-glycerophosphate, dexamethasone, ascorbic acid, Triton X-100, DMSO and Alizarin Red S were purchased from Sigma-Aldrich (St. Louis, MO, USA). Anti- $\beta$-actin (sc-47778), anti-p-extracellular signalregulated kinase (ERK; sc-377400), anti-t-ERK (sc-514302), anti-Runt-related transcription factor 2 (Runx2; sc-12488), anti-osteonectin (sc-13326) and anti-ColA1 (sc-59954) were purchased from Santa Cruz Biotechnology, Inc. (Santa Cruz, CA, USA). Alizarin Red was obtained from Sigma-Aldrich. An enhanced chemiluminescence (ECL) kit was purchased from Pierce Biotechnology/Thermo Fisher Scientific (Rockford, IL, USA). Alkaline phosphatase assay enzyme-linked immunosorbent assay (ELISA) kit was purchased from Abcam (Cambridge, MA, USA). All water used was glass distilled.

Ethics statement. The research was approved by the Ethics Committee of the Medical Ethics Committee of Guizhou Orthopedics Hospital. All experimental procedures were conducted in conformity with institutional guidelines for the care and use of laboratory animals.

Cell culture. Mesenchymal stem cells (MSCs) were collected from SD rats by flushing the femurs with $\alpha$-MEM supplemented with $10 \%$ FBS, $100 \mathrm{U} / \mathrm{ml}$ penicillin, and $100 \mu \mathrm{g} / \mathrm{ml}$ streptomycin. The obtained suspension was centrifuged at $300 \mathrm{x}$ g for $5 \mathrm{~min}$. The pellet was incubated in $5 \% \mathrm{CO}_{2}$ at $37^{\circ} \mathrm{C}$ for $72 \mathrm{~h}$. Hemopoietic cells are unable to adhere to the surface of culture dishes, while MSCs are able to adhere. Thus, we used this method to separate the cells. The primary cells were passaged, after 6 days from isolation. Adherent cells were subcultured using $0.2 \%$ trypsin plus, when MSCs reached $80 \%$ confluency.

Osteogenic differentiation. Cells were seeded into $10-\mathrm{cm}$ culture dishes or 24-well plates. For osteogenic differentiation, the cells were treated with osteogenic medium consisting of phenol red-free $\alpha$-MEM, $10 \%$ FBS, ascorbic acid $(50 \mu \mathrm{g} / \mathrm{ml})$, dexamethasone $\left(10^{-8} \mathrm{M}\right)$, and $\beta$-glycerophosphate $\left(10^{-2} \mathrm{M}\right)$. For the control, the cells were treated with normal medium consisting of $\alpha$-MEM supplemented with $10 \%$ FBS. The medium was changed twice every week.

ALP detection. ALP activity in the lysate was detected with the use of an alkaline phosphatase ELISA kit (Abcam) according to the manufacturer's instructoins. The absorbance was measured at a wavelength of $405 \mathrm{~nm}$ using a microplate reader (EL309; Bio-Tek, Winooski, VT, USA). The corresponding activity was calculated based on a standard curve according to a sample absorbance value.

Mineralization analysis. The intracellular calcium deposition was assessed by Alizarin Red staining. Briefly, MSCs were seeded into 12-well plates and grown under osteogenic conditions as described above. Control cells were treated with normal medium consisting of $\alpha$-MEM supplemented with $10 \%$ FBS. The medium was changed twice every week. At different culture days, the cells were fixed with ice-cold $70 \%$, washed with sterile 1X phosphate-buffered saline (PBS), stained with $2 \%$ (w/v) Alizarin Red S and visualized using a light microscope (SZX10; Olympus, Tokyo, Japan).

Plasmid construction and transfection. MicroRNAs mimicking miR-9 and miR-10 were obtained from GenePharma (Shanghai, China). Overexpression plasmids were purchased from Addgene (Cambridge, MA, USA). The cells were seeded into 6-well plates and incubated for $24 \mathrm{~h}$ to reach $90-100 \%$ confluency. Then, the cells were transfected using $\alpha$-MEM containing $5 \mu \mathrm{l}$ Lipofectamine and $100 \mathrm{nM}$ pre-miRs/well in a final volume of $1 \mathrm{ml}(500 \mu \mathrm{l})$ for $4 \mathrm{~h}$, and then replaced with growth medium and cultured for $48 \mathrm{~h}$. All experiments were performed for at least three biological repeats.

RNA extraction and RT-PCR. Total RNA was isolated from the cells using TRIzol reagent (Invitrogen). The RNA concentration was determined using a NanoDrop dispositive (Thermo Scientific, Waltham, MA, USA). For the RT-PCR assays, reverse transcription (RT) reactions for the miRNAs were carried out using stem-loop primers. The RT for miRNA-9 and miR-10 was carried out with $100 \mathrm{ng}$ of total RNA using thermostable M-MLV Reverse Transcriptase (Invitrogen). PCR reactions were performed with a Gene Amp PCR System 9700 (PerkinElmer, Waltham, MA, USA) and amplified for 35 cycles. The amplified products were separated by electrophoresis on a $2 \%$ agarose gel and visualized by ethidium bromide staining. Each product was visualized after separation using glyceraldehyde 3-phosphate dehydrogenase (GAPDH) as an internal control. Image density was quantified with a FluoroImager SI (Amersham Pharmacia Biotech, Amersham, UK).

For quantitative PCR assays, RT reactions for the miRNAs were performed using the miRNA First-Strand cDNA Synthesis kit (Invitrogen) following the manufacturer's instructions. Real-time PCR was performed using $1 \mu \mathrm{l}$ of cDNA in a $25 \mu 1$ reaction volume with LightCycler Real-time PCR system (Roche Applied Science, Indianapolis, IN, USA). The temperature profile of the reaction was $95^{\circ} \mathrm{C}$ for $15 \mathrm{~min}$, 40 cycles of denaturation at $95^{\circ} \mathrm{C}$ for $30 \mathrm{sec}$, annealing at $54^{\circ} \mathrm{C}$ and $58^{\circ} \mathrm{C}$ (miR-9 and -10) for $30 \mathrm{sec}$, and extension at $72^{\circ} \mathrm{C}$ for $1 \mathrm{~min}$. The relative expression levels were respectively normalized to GAPDH.

Western blot analysis. Briefly, nuclear and cytosolic extracts or total cell lysates were prepared and equal amounts of protein samples from the MSCs were subjected to sodium dodecyl sulfate-polyacrylamide gel electrophoresis (SDS-PAGE) using $10 \%$ gradient Tris-glycine gels. After that, the proteins were transferred to polyvinylidene difluoride (PVDF) membranes (Millipore, Temecula, CA, USA). Then, the blots were incubated with the following primary antibodies, after blocking with $5 \%$ fat-free milk for $1 \mathrm{~h}$. The primary antibodies used in the present study were as follows: osteonectin (1:400), ColA1 (1:400), Runx2 (1:400), t-ERK (1:800), p-ERK (1:800) and $\beta$-actin $(1: 1,000)$. The membranes were then incubated with horseradish peroxidase-conjugated secondary antibodies (sc-2073; Santa Cruz Biotechnology, Inc.) overnight at $4^{\circ} \mathrm{C}$. 
A

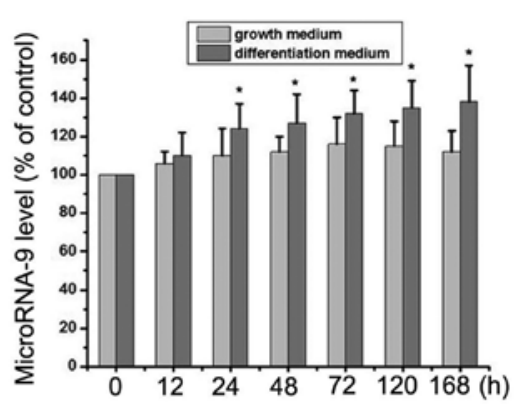

$\mathrm{B}$

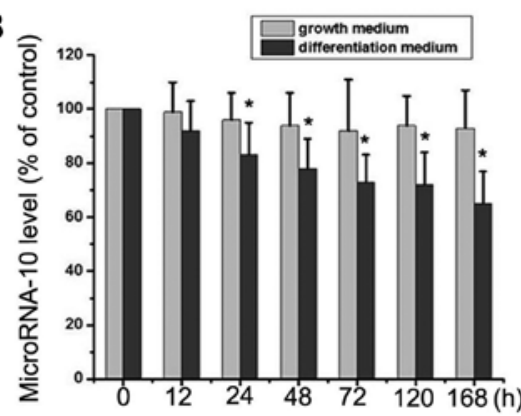

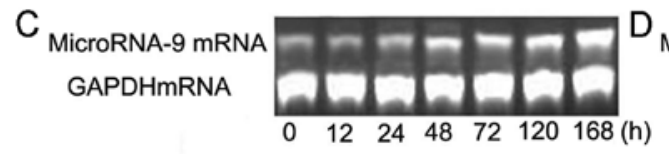
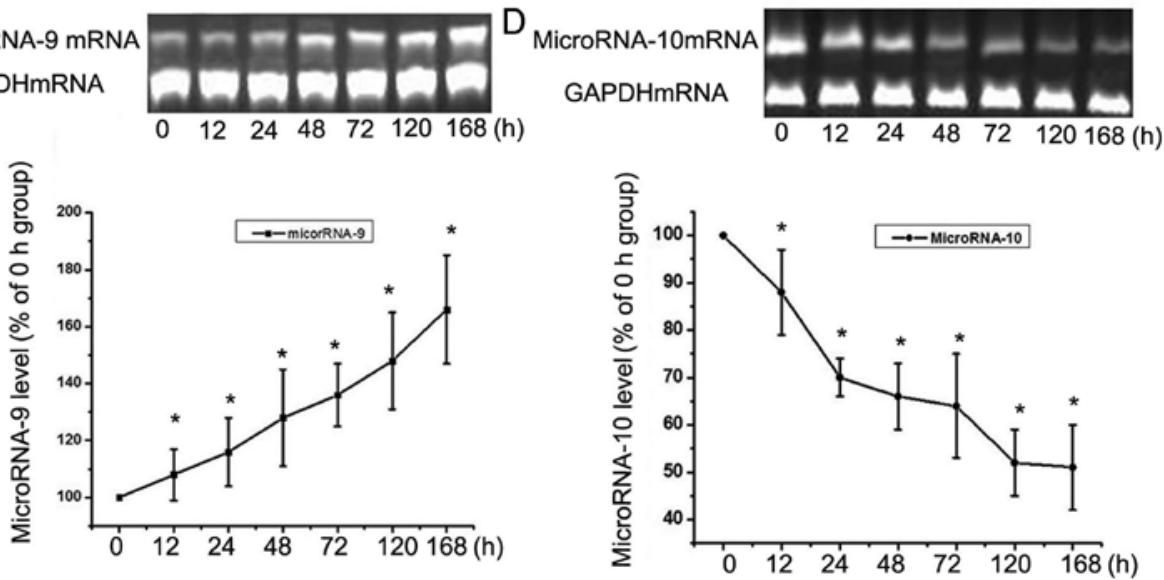

Figure 1. miR-9 and miR-10 expression during the process of osteogenic differentiation of bone mesenchymal stem cells (BMSCs). BMSCs were treated with growth medium or differentiation medium for several hours to investigate the changes in miR-9 and miR-10 expression by qRT-PCR. (A) miRNA-9 expression was increased along a temporal axis in BMSCs with differentiation medium. miR-9 expression was not significantly changed in BMSCs with growth medium. Data are expressed as the means $\pm \mathrm{SD},{ }^{*} \mathrm{p}<0.05$ growth medium vs. differentiation medium. (B) miRNA-10 expression was decreased along a temporal axis in BMSCs with differentiation medium. miRNA-10 expression was not significantly changed in BMSCs with growth medium. Data are expressed as the means $\pm \mathrm{SD},{ }^{*} \mathrm{p}<0.05$ growth medium vs. differentiation medium. (C) BMSCs were treated with differentiation medium for several hours and the expression of miR-9 was detected by RT-PCR. Data are expressed as the means \pm SD, ${ }^{*}$ p $<0.05$ vs. 0 h. Data show that miR- 9 was upregulated in a time-dependent manner. (D) BMSCs were treated with differentiation medium for several hours and the expression of miR-10 was detected by RT-PCR. Data are expressed as the means $\pm \mathrm{SD},{ }^{*} \mathrm{p}<0.05$ vs. $0 \mathrm{~h}$. Data show that miR-10 was downregulated in a time-dependent manner. miR, microRNA.

Protein detection was performed using an ECL kit. The relative amounts of various proteins were analyzed. The results were quantified by Quantity One software.

Statistical analysis. Data are reported as the means \pm SD and were analyzed using SPSS 17.0 (SPSS, Inc., Chicago, IL, USA) statistical software. Variance was homogenous for use of standard ANOVA methodology. After statistical significance was established by ANOVA, individual comparisons were made using Tukey's multiple comparison test. The level of significance was defined as $\mathrm{P}<0.05$.

\section{Results}

The changes in miR-9 and miR-10 expression during the process of osteogenic differentiation. Osteogenic differentiation can be regulated by multiple signaling molecules and transcriptional regulators, yet the role of miR-9 and miR-10 in the osteogenic differentiation of BMSCs is not clear. This study aimed to elucidate the role of miR-9 and miR-10 in the osteogenic differentiation of BMSCs. From the results of Fig. 1A and B, we found that, in the differentiation medium, the miR-9 expression was increased in a time-dependent manner while the expression of miR-10 was decreased in the differenti- ation medium during the process of osteogenic differentiation. However, in the normal growth culture medium, the miR-9 and miR-10 expression did not change significantly (Fig. 1). To further confirm the miR-9 and miR-10 changes in osteogenic induction, RT-PCR was conducted (Fig. 1C and D) and the results were in good agreement with the RT-qPCR results (Fig. 1A and B). Through the above study, we draw the following conclusions, that during the process of osteogenic differentiation, the expression of miR-9 was upregulated and miR-10 was downregulated as the culture time was prolonged.

The expression changes of miR-9 and miR-10 in BMSCs after transfection of the overexpression plasmids during the process of osteogenic differentiation. During the process of osteogenic differentiation, the expression of miR-9 and miR-10 were respectively upregulated and downregulated. Therefore, we aimed to ascertain whether the overexpression of miR-9 and miR-10 will alter the process of osteogenic differentiation in the BMSCs. RT-PCR analysis was performed from isolated total RNA for miR-9 and miR-10 levels after the cells were transfected with a plasmid expressing the corresponding gene at different times (Fig. 2A-D). Following transfection of the cells, the expression levels of miR-9 and miR-10 were significantly increased and peaked at $72 \mathrm{~h}$. Then we determined 

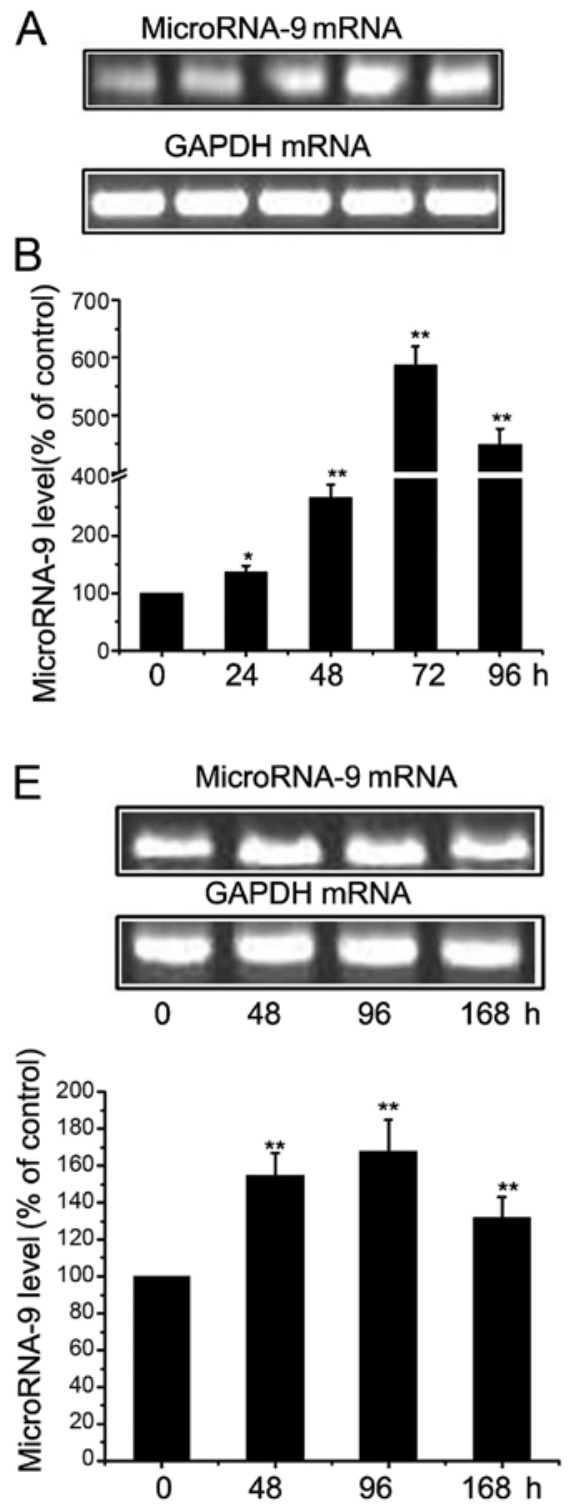
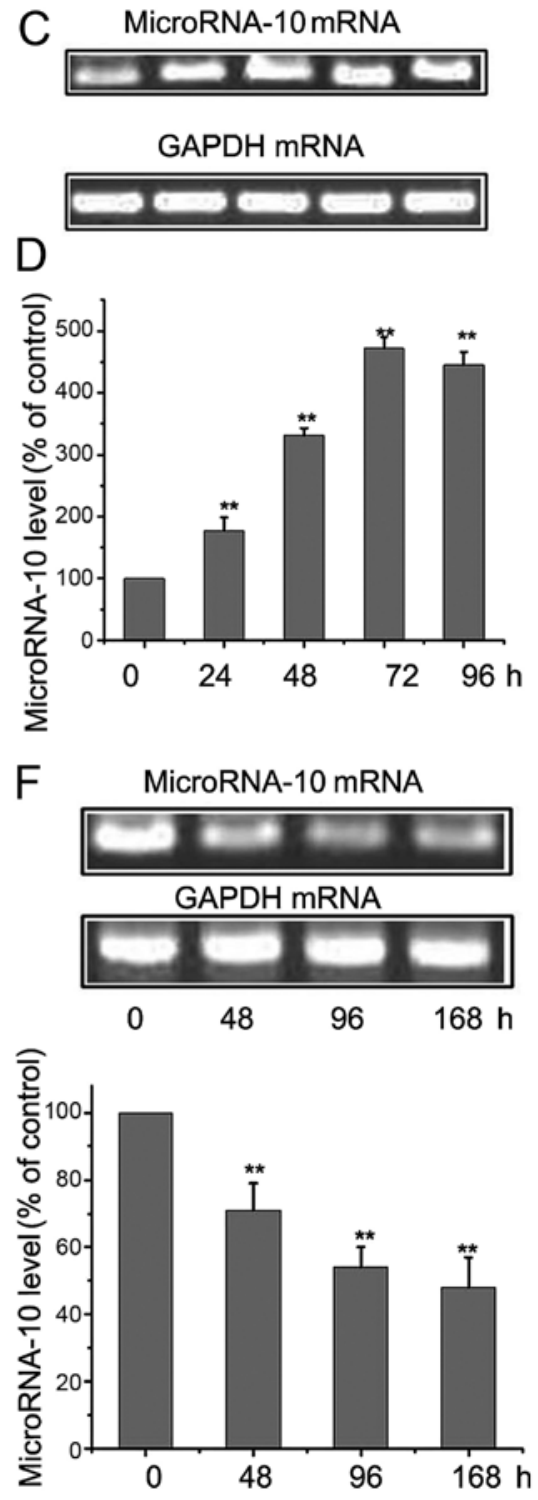

Figure 2. miR-9 and miR-10 expression during the process of osteogenic differentiation in bone mesenchymal stem cells (BMSCs) transfected with the miR-9 and miR-10 overexpressing plasmids (A) RT-PCR analysis was performed from isolated total RNA for miR-9 mRNA after miR-9 overexpression plasmid transfection at different times. (B) Values represent mean $\pm \mathrm{SD}$, of three independent experiments performed. GAPDH was used as loading control ("P $<0.05$ vs. $0 \mathrm{~h}$ group; ${ }^{* *} \mathrm{P}<0.01 \mathrm{vs.} 0 \mathrm{~h}$ group). (C) RT-PCR analysis was performed from isolated total RNA for miR-10 mRNA after miR-10 overexpression plasmid transfection at different times. Values represent mean \pm SD, of three independent experiments performed. GAPDH was used as loading control. (D) Values represent mean $\pm \mathrm{SD}$, of three independent experiments performed. GAPDH was used as loading control ( ${ }^{* *} \mathrm{P}<0.01 \mathrm{vs} .0 \mathrm{~h}$ group). (E) miR-9-overexpressing cells were treated with differentiation medium for $0,48,96$ and $168 \mathrm{~h}$ and the expression of miR-9 mRNA was detected by RT-PCR. Values represent mean \pm SD of three independent experiments performed. GAPDH was used as loading control (** $\mathrm{P}<0.01 \mathrm{vs}$. $0 \mathrm{~h}$ group). (F) miR-10-overexpressing cells were treated with differentiation medium for $0,48,96$ and $168 \mathrm{~h}$ and the expression of miR- 9 mRNA was detected by RT-PCR. Values represent mean \pm SD of three independent experiments performed. GAPDH was used as loading control (" $\mathrm{P}<0.01$ vs. $0 \mathrm{~h}$ group). miR, microRNA.

changes in miR-9 and miR-10 expression (after transfection with the plasmids for $72 \mathrm{~h}$ ) in differentiation medium at 0,48 , 96 and 168 h. As shown in Fig. 2E and $F$ the expression of miR-9 was upregulated and peeked at $96 \mathrm{~h}$, while expression of miR-10 was downregulated at $96 \mathrm{~h}$ and reached a minimum at $168 \mathrm{~h}$. It can be seen that during the process of osteogenic differentiation, there are changes in miR-9 and miR-10 expression. We consider that these two genes will have an effect on osteogenesis.

The effects of miR-9 and miR-10 on osteogenic differentiation of BMSCs. To investigate the role of miR-9 and miR-10 in the osteogenic differentiation of BMSCs, the overexpression plasmid transfection was employed to alter the gene expression in our study. We treated both miR-9- and miR-10-overexpressing cells and control cells were treated with differentiation medium for 7 days, and various osteogenic differentiation indicators were detected. As shown in Fig. 3A, mineralization was noted in the BMSCs on day 7 after treatment with the different vectors. Overexpression of miR-9 markedly increased the mineralization effects compared with the control group, while overexpression of miR-10 diminished the mineralization. Moreover, in the miRNA-10-overexpressing BMSC group, osteonectin and ColA1 protein levels were significantly inhibited, while miR-9 increased the levels, compared to the control group. Furthermore, in the miR-9-overexpressing 


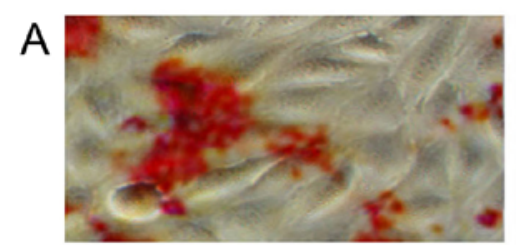

control

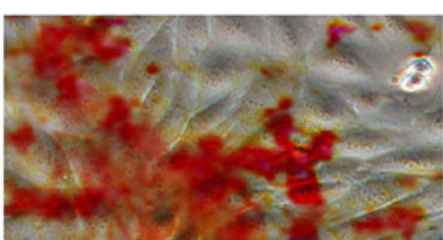

MicroRNA-9

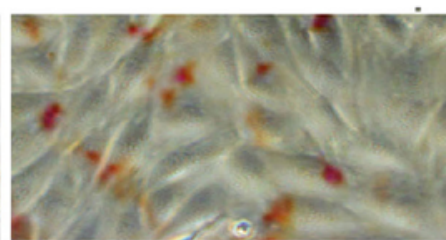

MicroRNA-10

C

ColA1

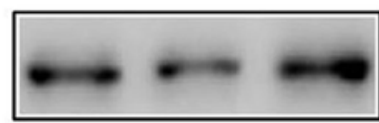

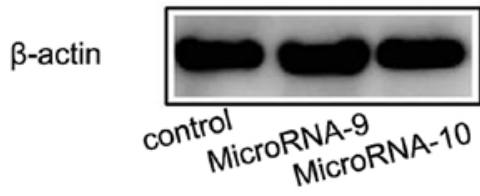

$\beta$-actin

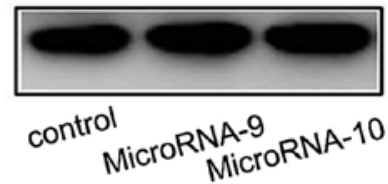

혼
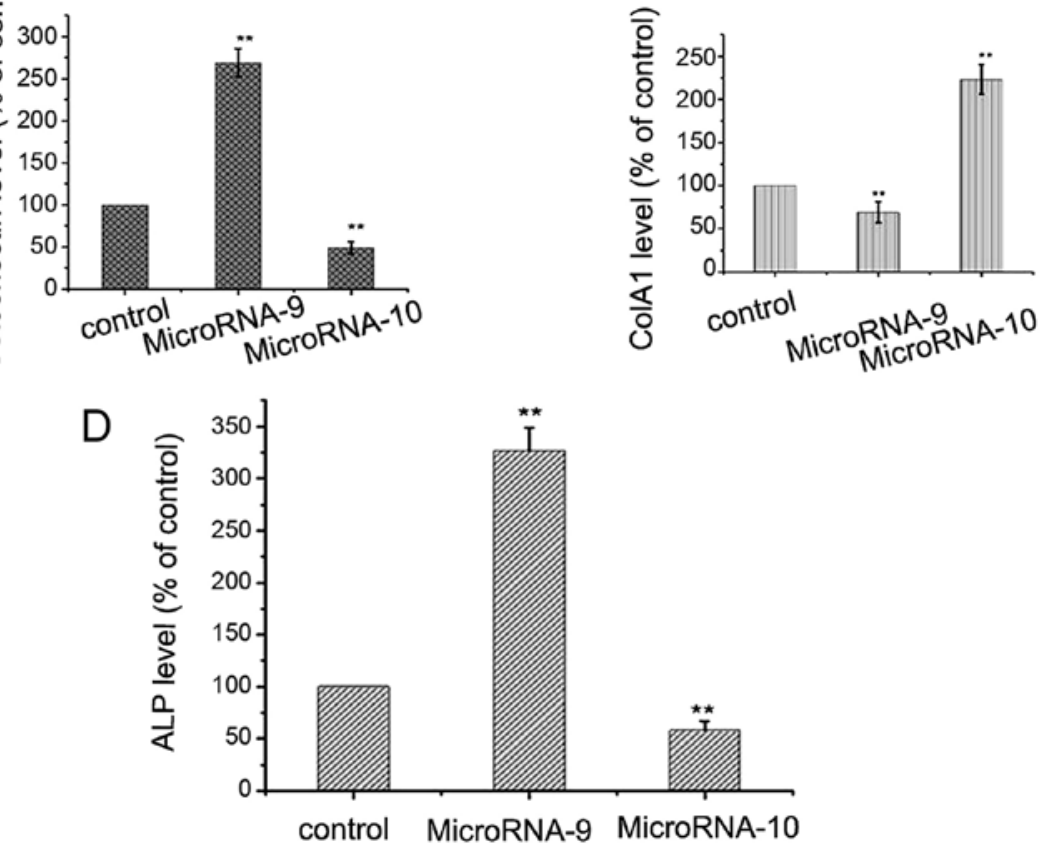

Figure 3. Role of miR-9 and miR-10 in the osteogenic differentiation of bone mesenchymal stem cells (BMSCs). (A) miR-9- and miR-10-overexpressing cells and control cells were treated with differentiation medium for 7 days, and mineralization was detected in the BMSCs. (B) Cells were treated as described above, and the expression of osteonectin was detected by western blotting. Values represent the mean \pm SD of three independent experiments performed. $\beta$-actin was used as loading control (** $\mathrm{P}<0.01$ vs. control). (C) Cells were treated as described above, and the expression of ColA1 was detected by western blotting. Values represent the mean $\pm \mathrm{SD}$ of three independent experiments performed. $\beta$-actin was used as loading control $\left({ }^{* *} \mathrm{P}<0.01 \mathrm{vs}\right.$. control). (D) $\mathrm{Cells}$ were treated as described above, and the expression of ALP was detected by ELISA. Values represent mean \pm SD of three independent experiments performed. (** $\mathrm{P}<0.01$ vs. control). miR, microRNA

BMSCs, the alkaline phosphatase (ALP) activity levels were increased, while it is decreased in the miR-10-overexpressing BMSCs (Fig. 3D). These results illustrate that miR-9 promotes osteogenic differentiation while miR-10 inhibits osteogenic differentiation.

miR-9 and miR-10 activate or inhibit Runx 2 expression and ERK signaling pathway. Previous studies have found that the transcription factor, Runx 2 is one of the earliest and most specific markers of osteogenesis (13). We next explored the effects of miR-9 and miR-10 on Runx 2 expression. As shown in Fig. 4A, overexpression of miR-9 increased protein expression of Runx2, while the overexpression of miR-10 decreased the level of Runx 2 by western blot analysis. Moreover, extracellular signal-regulated kinase (ERK) and mitogen-activated protein kinase (MAPK) signaling pathways have widely recognized roles in osteogenic differentiation (14). Thus, we aimed to ascertain whether miR-9 and miR-10 regulate osteogenic differentiation via these pathways. As shown in Fig. 4B and C, overexpression of miR-9 upregulated the level of phosphorylated ERK, while miR-10 downregulated the level of p-ERK. Notably, the total level of ERK did not change significantly regardless of whether the cells were transfection or not with miR-9 and miR-10. Our results imply that miR-10 negatively regulates osteogenic differentiation via ERK/MPAK signaling and miR-10 positively activates this signaling pathway. 
A

Runx2

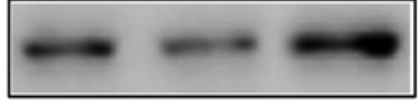

$\beta$-actin
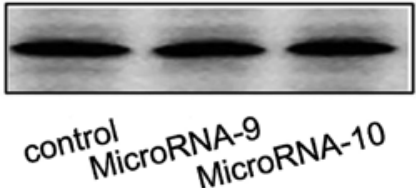

B
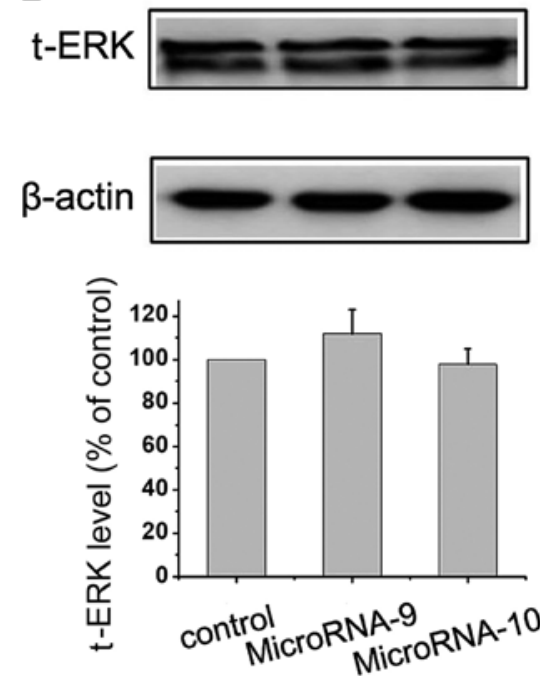

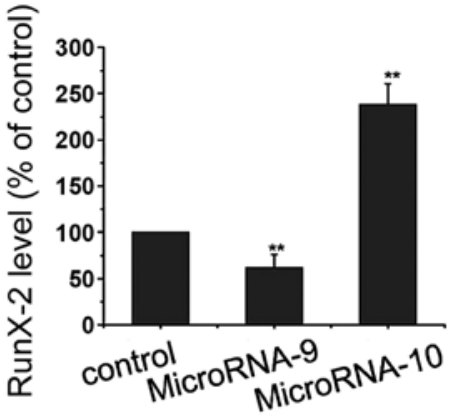

C
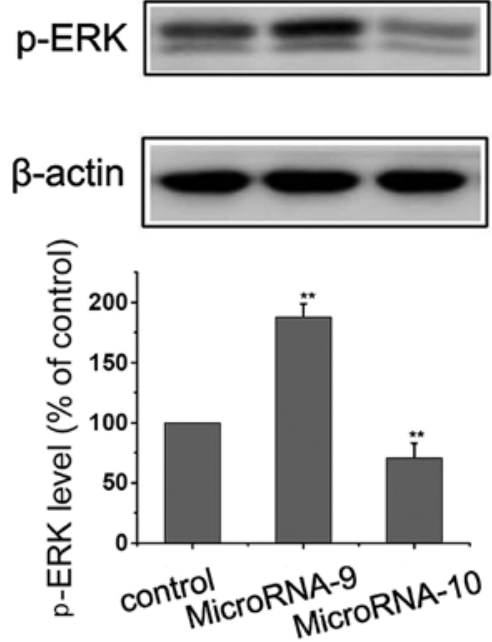

Figure 4. Effects of miR-9 and miR-10 on the Runt-related transcription factor 2 (Runx2) expression and extracellular signal-regulated kinase (ERK) signaling pathway in the osteogenic differentiation of bone mesenchymal stem cells (BMSCs). (A) miR-9- and miR-10-overexpressing cells and control cells were treated with differentiation medium for 7 days, and the expression of Runx-2 was detected by western blotting. Values represent mean \pm SD of three independent experiments performed ( ${ }^{* *} \mathrm{P}<0.01 \mathrm{vs}$. control). (B) Cells were treated as described above, and the expression of $\mathrm{t}-\mathrm{ERK}$ was detected by western blotting. Values represent the mean \pm SD of three independent experiments performed. $\beta$-actin was used as loading control. (C) Cells were treated as described above, and the expression of p-ERK was detected by western blotting. Values represent the mean \pm SD of three independent experiments performed. $\beta$-actin was used as loading control ( ${ }^{* *} \mathrm{P}<0.01$ vs. control).

\section{Discussion}

miRNAs are short non-coding small RNAs and are highly conserved across different species as well as highly specific for developmental stages (15). Since miRNAs are key regulators of gene expression through the post-transcriptional and posttranslational modification of mRNAs, studies have focused on the impact of miRNAs on cell growth, differentiation and apoptosis and have demonstrated the biosynthesis, function and the molecular regulation of miRNAs in normal physiological and pathological conditions in different diseases $(16,17)$. Through the binding of the 3'-UTR of target mRNAs, miRNAs induce the silencing of the protein expression of target genes through translational repression or mRNA degradation (18).

Recently, a number of studies have revealed that miRNAs participate in bone metabolism, including osteoblast growth, differentiation and stem cell osteogenic differentiation, which have shed light on the mechanisms underlying osteogenic differentiation $(19,20)$. Previous studies have also found that during the process of differentiation of BMSCs towards adipogenic, osteogenic and chondrogenic lineages, the expression levels of miR-196a, miR-378-star, miR-486-5p and miR-664 were upregulated; miR-708, miR-3197 and miR-637 were downregulated in elderly subjects (ranging from 65 to 80 years of age) compared with young subjects (ranging from 17 to 30 years of age) $(10,21,22)$. In recent years, miR-9 was reported as having an important role in tumor tumorigenesis and the progression of numerous types of cancers, while our studies have found that miR-9 may be a key mediator in osteogenic differentiation $(23,24)$. Similarly, miR-10 is co-expressed with a set of Hox genes and has been found to regulate the translation of Hox transcripts and attracted attention $(25,26)$. Bioinformatic data also revealed that miR-10 could bind with many bone metabolism-related mRNAs, which indicates that the function of miR-10 is more complex than expected. In the present study, we first detected the expression of miR-9 and miR-10 in MSCs with nomal growth medium and osteogenic differentiation medium, and the results showed that miR-9 and miR-10 expression was slightly altered in a normal growth status, while they were significantly altered under osteogenic differentiation conditions with upregulation of miR-9 and downregulation of miR-10. Moreover, overexpression of miR-9 promoted osteogenic differentiation while overexpression of miR-10 suppressed it.

Runx 2 as an osteogenesis-specific transcription factor plays an important role in the osteogenesis of MSCs not 
only promoting the expression of osteogenesis-related genes, improving the bone microenvironment but also having a close association with a large number of signaling molecules inc luding TGF- $\beta$, BMP, Wnt, Hedgehog, FGF and microRNAs $(27,28)$. Our data found that miR-9 and miR-10 influence the expression of Runx2, with upregulation by miR-9 and downregulation by miR-10 in good agreement with previous studies. Moreover, previous studies have indicated that the MAPK signaling pathway may be involved in the osteogenic differentiation of BMSCs and Runx 2 is physiologically regulated by the MAPK pathway $(29,30)$. In this study, we found that altering the expression of miR-9 and miR-10 significantly influenced the expression of Runx and the phosphorylation of ERK during BMSC osteogenic differentiation.

In conclusion, our findings revealed that miR-9 positively regulated the expression of the osteogenesis-related Runx2 gene and overexpression of miR-9 promoted osteogenic differentiation of BMSCs. By contrast, miR-10 overexpression not only inhibited the expression of Runx 2 but also negatively regulated osteogenic differentiation. Nonetheless, further experiments are required to show a correlation between miR-9 and miR-10 expression and osteogenic differentiation of BMSCs in our study. In the future, miRNAs may serve as crucial regulators of osteogenic differentiation.

\section{References}

1. Curtis EM, Moon RJ, Dennison EM, Harvey NC and Cooper C: Recent advances in the pathogenesis and treatment of osteoporosis. Clin Med (Lond) 15 (Suppl 6): s92-s96, 2015.

2. Piscitelli P, Iolascon G, Gimigliano F, Gimigliano A, Marinelli A, Di Nuzzo R, Colì G, Di Paola L, Gianicolo E, Chitano G, et al: Osteoporosis and cardiovascular diseases' cosegregation: Epidemiological features. Clin Cases Miner Bone Metab 5: 14-18, 2008.

3. Wang Y, Tao Y, Hyman ME, Li J and Chen Y: Osteoporosis in china. Osteoporos Int 20: 1651-1662, 2009.

4. Lin X, Xiong D, Peng YQ, Sheng ZF, Wu XY, Wu XP, Wu F, Yuan LQ and Liao EY: Epidemiology and management of osteoporosis in the People's Republic of China: Current perspectives. Clin Interv Aging 10: 1017-1033, 2015.

5. Geusens P: New insights into treatment of osteoporosis in postmenopausal women. RMD Open 1 (Suppl 1): e000051, 2015.

6. Xie C, Jin J, Lv X, Tao J, Wang R and Miao D: Anti-aging effect of transplanted amniotic membrane mesenchymal stem cells in a premature aging model of Bmi-1 deficiency. Sci Rep 5: 13975, 2015.

7. Ma L, Aijima R, Hoshino Y, Yamaza H, Tomoda E, Tanaka Y, Sonoda S, Song G, Zhao W, Nonaka K, et al: Transplantation of mesenchymal stem cells ameliorates secondary osteoporosis through interleukin-17-impaired functions of recipient bone marrow mesenchymal stem cells in MRL/lpr mice. Stem Cell Res Ther 6: 104, 2015.

8. Pittenger MF, Mackay AM, Beck SC, Jaiswal RK, Douglas R, Mosca JD, Moorman MA, Simonetti DW, Craig S and Marshak DR: Multilineage potential of adult human mesenchymal stem cells. Science 284: 143-147, 1999.

9. Bartel DP: MicroRNAs: Genomics, biogenesis, mechanism, and function. Cell 116: 281-297, 2004.

10. Fang S, Deng Y, Gu P and Fan X: MicroRNAs regulate bone development and regeneration. Int J Mol Sci 16: 8227-8253, 2015

11. Zhang GY, Wang J, Jia YJ, Han R, Li P and Zhu DN: MicroRNA-9 promotes the neuronal differentiation of rat bone marrow mesenchymal stem cells by activating autophagy. Neural Regen Res 10 314-320, 2015.
12. Varendi K, Kumar A, Härma MA and Andressoo JO: miR-1, miR-10b, miR-155, and miR-191 are novel regulators of BDNF. Cell Mol Life Sci 71: 4443-4456, 2014.

13. Shakibaei M, Shayan P, Busch F, Aldinger C, Buhrmann C, Lueders $C$ and Mobasheri A: Resveratrol mediated modulation of Sirt-1/Runx2 promotes osteogenic differentiation of mesenchymal stem cells: Potential role of Runx2 deacetylation. PLoS One 7: e35712, 2012

14. Wu Y, Zhou J, Li Y, Zhou Y, Cui Y, Yang G and Hong Y: Rap1A regulates osteoblastic differentiation via the ERK and p38 mediated signaling. PLoS One 10: e0143777, 2015.

15. Li Y and Sarkar FH: MicroRNA targeted therapeutic approach for pancreatic cancer. Int J Biol Sci 12: 326-337, 2016.

16. Kuninty PR, Schnittert J, Storm G and Prakash J: MicroRNA targeting to modulate tumor microenvironment. Front Oncol 6: 3, 2016.

17. Taylor MA and Schiemann WP: Therapeutic opportunities for targeting microRNAs in cancer. Mol Cell Ther 2: 1-13, 2014.

18. Ott CE, Grünhagen J, Jäger $M$, Horbelt $\mathrm{D}$, Schwill S, Kallenbach K, Guo G, Manke T, Knaus P, Mundlos S, et al: MicroRNAs differentially expressed in postnatal aortic development downregulate elastin via 3' UTR and coding-sequence binding sites. PLoS One 6: e16250, 2011.

19. Yan J, Guo D, Yang S, Sun H, Wu B and Zhou D: Inhibition of miR-222-3p activity promoted osteogenic differentiation of hBMSCs by regulating Smad5-RUNX2 signal axis. Biochem Biophys Res Commun 470: 498-503, 2016.

20. Weilner S, Skalicky S, Salzer B, Keider V, Wagner M, Hildner F, Gabriel C, Dovjak P, Pietschmann P, Grillari-Voglauer R, et al: Differentially circulating miRNAs after recent osteoporotic fractures can influence osteogenic differentiation. Bone 79: 43-51, 2015

21. Zhang JF, Fu WM, He ML, Wang H, Wang WM, Yu SC, Bian XW, Zhou J, Lin MC, Lu G, et al: MiR-637 maintains the balance between adipocytes and osteoblasts by directly targeting Osterix. Mol Biol Cell 22: 3955-3961, 2011.

22. Trompeter HI, Dreesen J, Hermann E, Iwaniuk KM, Hafner M, Renwick N, Tuschl T and Wernet P: MicroRNAs miR-26a, miR-26b, and miR-29b accelerate osteogenic differentiation of unrestricted somatic stem cells from human cord blood. BMC Genomics 14: 111, 2013.

23. Sun J, Fang K, Shen H and Qian Y: MicroRNA-9 is a ponderable index for the prognosis of human hepatocellular carcinoma. Int J Clin Exp Med 8: 17748-17756, 2015.

24. Drakaki A, Hatziapostolou M, Polytarchou C, Vorvis C, Poultsides GA, Souglakos J, Georgoulias V and Iliopoulos D: Functional microRNA high throughput screening reveals miR-9 as a central regulator of liver oncogenesis by affecting the PPARA-CDH1 pathway. BMC Cancer 15: 542, 2015.

25. Lund AH: miR-10 in development and cancer. Cell Death Differ 17: 209-214, 2010.

26. Woltering JM and Durston AJ: MiR-10 represses HoxB1a and HoxB3a in zebrafish. PLoS One 3: e1396, 2008.

27. Xu J, Li Z, Hou Y and Fang W: Potential mechanisms underlying the Runx2 induced osteogenesis of bone marrow mesenchymal stem cells. Am J Transl Res 7: 2527-2535, 2015.

28. Choung HW,Lee DS, Lee HK, Shon WJ and Park JC: Preameloblastderived factors mediate osteoblast differentiation of human bone marrow mesenchymal stem cells by Runx2-osterix-BSP signaling. Tissue Eng Part A 22: 93-102, 2016.

29. Salasznyk RM, Klees RF, Hughlock MK and Plopper GE: ERK signaling pathways regulate the osteogenic differentiation of human mesenchymal stem cells on collagen I and vitronectin. Cell Commun Adhes 11: 137-153, 2004.

30. Saito T, Nishida K, Furumatsu T, Yoshida A, Ozawa M and Ozaki T: Histone deacetylase inhibitors suppress mechanical stress-induced expression of RUNX-2 and ADAMTS-5 through the inhibition of the MAPK signaling pathway in cultured human chondrocytes. Osteoarthritis Cartilage 21: 165-174, 2013. 